\title{
High-power UV-LED degradation: Continuous and cycled working condition influence
}

\author{
F.J. Arques-Orobon ，N. Nuñez ，M. Vazquez ，C. Segura-Antunez ，V. González-Posadas
}

\begin{abstract}
A B S T R A C T
High-power (HP) UV-LEDs can replace UV lamps for real-time fluoro-sensing applications by allowing portable and autonomous systems. However, HP UV-LEDs are not a mature technology, and there are still open issues regarding their performance evolution over time. This paper presents a reliability study of 3 W UV-LEDs, with special focus on LED degradation for two working conditions: continuous and cycled ( $30 \mathrm{~s}$ ON and $30 \mathrm{~s}$ OFF). Accelerated life tests are developed to evaluate the influence of temperature and electrical working conditions in high-power LEDs degradation, being the predominant failure mechanism the degradation of the package. An analysis that includes dynamic thermal and optical HP UV-LED measurements has been performed. Static thermal and stress simulation analysis with the finite element method (FEM) identifies the causes of package degradation. Accelerated life test results prove that HP UV-LEDs working in cycled condition have a better performance than those working in continuous condition.
\end{abstract}

\section{Introduction}

The main applications for ultraviolet (UV) light sources are in the graphic arts market, water purification and UV ink curing. UV lamps are also used as light sources in complex spectroscopy fluoro-sensing techniques, which analyze materials or molecules diluted in liquids $[1,2]$. The HP UV-LED will be a relevant improvement in this area [3].

To produce the effect of fluorescence, it is necessary that the substance to be detected emits at a wavelength greater than the light source used. Therefore, UV light sources are required to detect hydrocarbon pollutants in water. UV light sources must emit sufficient light so that the spectrometer can detect the pollutant's pattern. During the last several years, the development of high-power (HP) ultraviolet (UV) light emitting diodes (LEDs) has improved very quickly, and currently, HP UV-LEDs are commercially available from different manufacturers. In previous works [4], we analyzed the viability and advantages of using UV-LEDs instead of conventional UV lamps for fluorescence applications due to their size, response time, power consumption, heat dissipation and cost.
These advantages could help to develop a new generation of portable and autonomous fluoro-sensing equipment $[4,5]$ for hydrocarbon real-time detection in inland waters [4]. Another expected advantage that must be analyzed is the lifetime and degradation of the UV light source. A conventional UV light source has an estimated life of approximately $500-2000 \mathrm{~h}$, while the UV-LED estimated life is $20,000-50,000 \mathrm{~h}$ [6]. This is an important issue because using HP UV-LEDs in real-time pollutant fluorescence detection [4], it is necessary to ensure the maintenance of performance during a continuous ( $24 \mathrm{~h} /$ day) and long period of working time. Therefore, a careful reliability analysis with accelerated life tests (ALT) that is presented in this work is needed. The results could be applied for HP UV-LEDs in fluoro-sensing and other critical lighting systems that work during a long period of time. Thus, the main results and conclusions of this work could be extended to other technological areas with UV-LEDs.

The main objective of this work is to identify the influence of working conditions on the degradation of UV-LED light output. This paper has been organized in the following sections: First, the LED package and experimental setup in Section 2 will be described. Later, the influence of the proposed working conditions on the LEDs is evaluated. The core of this paper is the influence of working conditions on package degradation, which causes UV-LED failures. This paper ends with proposed improvements and conclusions in Section 7. 


\section{Experimental set up}

\subsection{LEDs and package description}

The main characteristics of these UV-LEDs are $3 \mathrm{~W}, 365-\mathrm{nm}$ wavelength, $700-\mathrm{mA}$ maximum nominal continuous current and an optical power of $900 \mathrm{~mW}$. The LEDs have been tested at $600-\mathrm{mA}$ current injection ( $85 \%$ of the maximum nominal continuous current).

An LED chip is encapsulated in a surface mount ceramic package with a glass lens filled with silicone (Figs. 1 and 2). The LED package has a footprint of approximately $4.5 \mathrm{~mm} \times 4.5 \mathrm{~mm}$ and is welded on a metal core printed circuit board (MCPCB), also known as an insulated metal substrate (IMS) type [7] with a standard star shape of $19.9 \mathrm{~mm}$ and an aluminum core (Fig. 2, left). In this structure, a heat sink of $40 \times 40 \times 18 \mathrm{~mm}$ with thermal tape $\left(\right.$ ALD $^{\mathrm{TM}}$, heat sink BGA-STD-115) has been screwed and adhered to the star MCPCB, as shown in Figs. 1 and 2.

Fig. 2 shows a high-power UV-LED that is mounted for the tests. The left figure shows the LED used with the star MCPCB, and the right figure shows the same device mounted with the heat sink. In this application, the LED is facing down because it has to perform the excitation of water with possible pollutants that is flowing through a duct; and for that, the fins of the heat sink look up for an adequate natural convection.

\subsection{Test description}

For this analysis, 11 high-power UV-LEDs have been characterized under ALTs during approximately $4000 \mathrm{~h}$ at three ambient temperatures $\left(60^{\circ} \mathrm{C}, 75^{\circ} \mathrm{C}\right.$ and $\left.90^{\circ} \mathrm{C}\right)$ under natural convection and two working conditions (continuous and low frequency cycled to $30 \mathrm{~s} \mathrm{ON}$ and $30 \mathrm{~s}$ OFF). This low frequency cycle has been selected instead of high frequency, i.e. $10 \mathrm{kHz}$, because the pollutant fluorescence pattern can be measured during continuously emitted light, making it possible to identify the hydrocarbon type. High frequency pulse light introduces a new variable of identification, the fluorescent decay, which makes the identification difficult. Other duty cycles with 60-s working periods have been considered, but $30 \mathrm{~s} \mathrm{ON}$ and $30 \mathrm{~s}$ OFF has been selected as the most suitable for this application (see the next section).

For each ALT, we used one power supply with precise current control. All LEDs have been connected in series at a constant current $(600 \mathrm{~mA})$ that does not vary during tests and a voltage that follows the sum of LED working point voltages during the test.

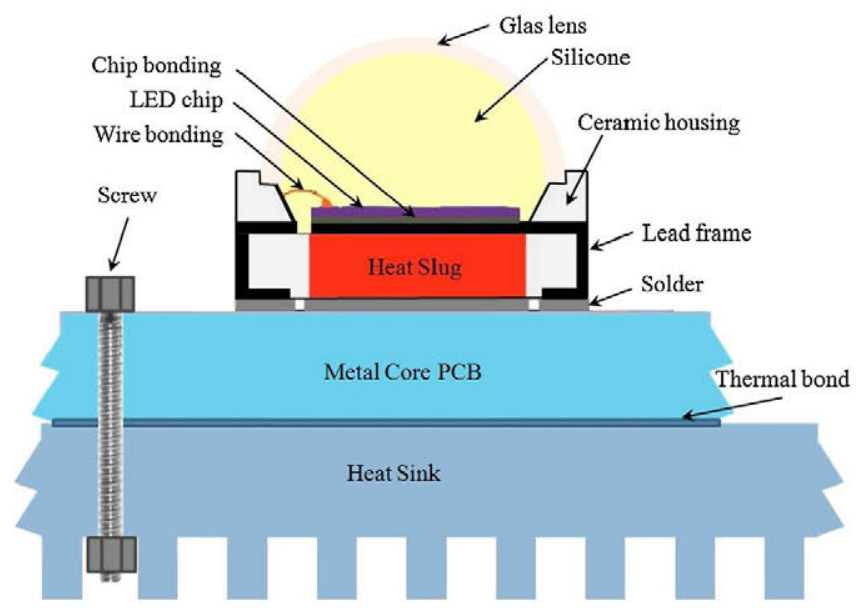

Fig. 1. UV-LED package with $M C P C B$ circuit and heat sink.

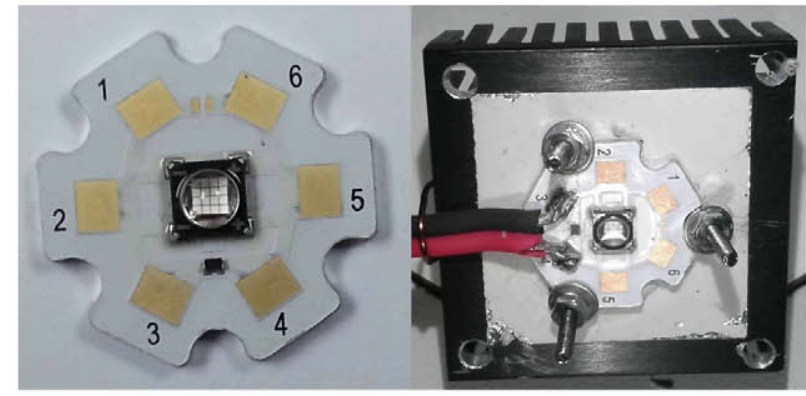

Fig. 2. Photograph of HP UV-LED over star MCPCB and assembled with heat sink.

For the working cycled condition, a custom circuit has been developed to control and avoid overcurrent peaks in commutations.

The performance parameter used to characterize the LEDs during the tests was light output. It is evaluated through a MIGHTEX ${ }^{\mathrm{TM}}$ spectrometer that measures the optical power spectrum. LEDS were also characterized by $I-V$ curves inside and outside the oven. Furthermore, package degradation has been evaluated by using optical microscopy. Additional information related to the instrumentation system used in this work has been described in a previous work [8].

\section{Working condition analysis}

LED density current and temperature are very important issues for high-power LEDs because LED light output usually increases with current and high-power LEDs work at high currents. However, high density currents expect an increase in temperature and both parameters, density current and temperature negatively affect LED degradation and reliability [9-13]. For that, it is necessary to trade-off among functionality, great luminosity with high current, and high reliability.

With the objective to reduce working temperature and the injected current working time, continuous working conditions and various cycled working conditions have been tested. The main objectives of this cycled working condition are to reduce the real working period over calendar time and to reduce the working temperature by maintaining the injected current and functionality. In cycled mode the calendar time includes the whole cycled time (ON time plus OFF time). On the other hand, the cycled conditions have an additional switch $\mathrm{ON}-\mathrm{OFF}$ stress that must be considered. Assessing the benefits and drawbacks of both working conditions is one of the objectives of this work. Therefore, two working conditions have been analyzed with equivalent functionality in calendar time:

- Continuous working condition. LEDs are working in a continuous condition during the entire period of time.

- Cycled working condition. LEDs are working with ON and OFF injected cycles for a period of $60 \mathrm{~s}$.

Before the temperature ALT, we evaluated the influence of both working conditions on LED junction temperature and LED light output. We have analyzed three cycled conditions: $10 \mathrm{~s}$ ON and $50 \mathrm{~s}$ OFF, $30 \mathrm{~s} \mathrm{ON}$ and $30 \mathrm{~s}$ OFF and $50 \mathrm{~s} \mathrm{ON}$ and $10 \mathrm{~s}$ OFF. These three working conditions are suitable for detecting pollutants in water by using the real-time fluorescence system and by reducing the LED working time and temperature. The results of the $50 \mathrm{~s}$ ON and $10 \mathrm{~s}$ OFF cycled working condition are very similar in temperature to the continuous condition and, therefore, will not be presented. 
The LED junction temperature at the different working conditions has been measured using the $I-V$ LED curve as a sensor of junction temperature [12,14-17]. Voltage at a prefixed low injected current is used to measure the LED junction temperature, Tj. Previously, a calibration between voltage at a low injected current and $\mathrm{Tj}$ was made in the range of the working temperature. In Fig. 3, the LED Tj evolution with time for the $25^{\circ} \mathrm{C}$ ambient temperature, $T_{a}$, and natural convection for the three working conditions are shown.

Simultaneous to the Tj measurements, the optical power spectrum was recorded. The light output maximum peak at $25^{\circ} \mathrm{C}$ is referenced with respect to its initial value in Fig. 4. From Figs. 3 and 4, it can be observed that as the LEDs start to work, the junction temperature increases and the light output decreases. During OFF states without light output, the junction temperature decreases.

From the analysis of Figs. 3 and 4, we can conclude that for the continuous working condition, the increase in junction temperature is significantly larger $\left(20.5^{\circ} \mathrm{C}\right)$ than in both cycled working conditions: a maximum of $14.5^{\circ} \mathrm{C}$ for $30 \mathrm{~s} \mathrm{ON}$ and $30 \mathrm{~s} \mathrm{OFF}$ and $10^{\circ} \mathrm{C}$ for $10 \mathrm{~s}$ ON and $50 \mathrm{~s}$ OFF. This junction temperature increase negatively affects the light output. In the continuous working condition, the light output reduction with respect to the initial value is larger $(10.2 \%)$ than in cycled working conditions (7.2\% and $5 \%$ ). Additionally, it can be observed that the time for stable functionality is lower than $30 \mathrm{~min}$.

One problem with the cycled working condition is the light output level. Thus, in Fig. 5, a detailed time graph of the normalized light output is represented. In the $30 \mathrm{~s}$ ON cycled condition, a relatively stabilized output can be observed during the last $10 \mathrm{~s}$ of the cycle that can be used for fluoro-sensing applications [4]. However, in the $10 \mathrm{~s}$ ON cycled mode, the stabilization is considerably poorer and makes the sensing use difficult; thus, it is dismissed. As observed in Figs. 3 and 5, two time constants are clearly involved in $\mathrm{Tj}$ for the $30 \mathrm{~s} \mathrm{ON}$ and $30 \mathrm{~s}$ OFF cycles and for the light output (see Fig. 5). The first vertical increase/decrease of $\mathrm{Tj}$ is due to the low thermal capacitance of the UV-LED chip and the ceramic package, and the slow slope corresponds with the set of star MCPCB and the heat sink.

Applying the analogy between thermal and electrical RC circuits and the procedure for the dynamic thermal analysis of [18], we have modeled the thermal circuit as an electrical equivalent circuit with two series RC networks (R1C1 for the LED package and $\mathrm{R} 2 \mathrm{C} 2$ for the aluminum sinks) (Fig. 6). The sum of the thermal resistance can be obtained from the power dissipated during the ON state of $1.49 \mathrm{~W}$ (2.14 W of LED power less $0.65 \mathrm{~W}$ of emitted light) and the average stable increment of temperature $\left(11.32^{\circ} \mathrm{C}\right)$. This results in $R \mathrm{th}_{j-a}=15.2^{\circ} \mathrm{C} / \mathrm{W} \quad(R 1+R 2=15.2 \Omega$ in the electrical circuit $)$. Fitting the measured decrement of temperature to the sum of the discharge of two $R C$ circuits $(V(R 1)+V(R 2)+25 \mathrm{~V})$, the constants

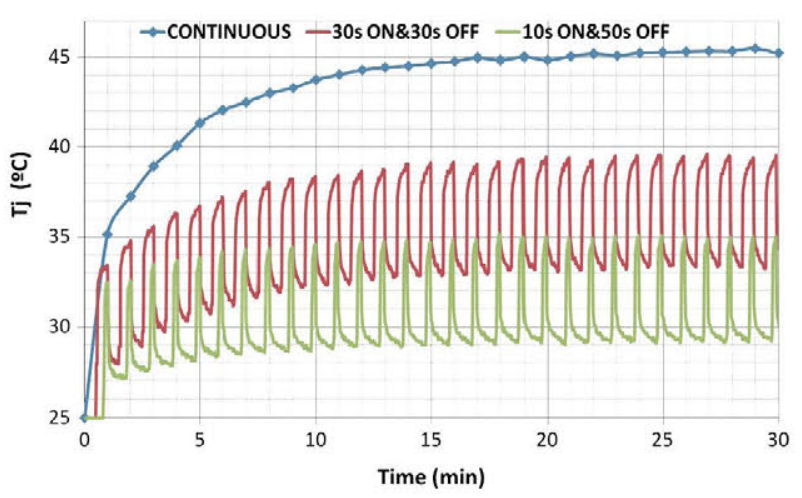

Fig. 3. LED junction temperature Tj vs. time at three working conditions.

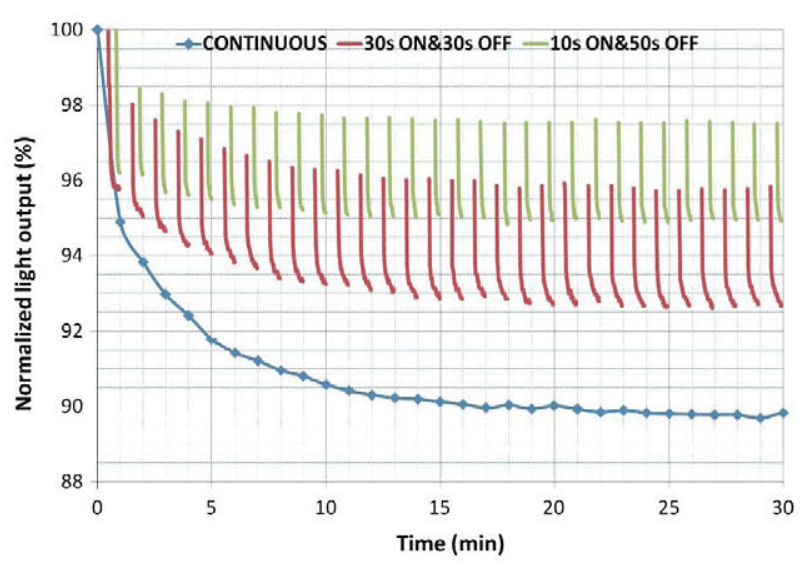

Fig. 4. Normalized light output with respect to the initial instant of time vs. time at three working conditions.

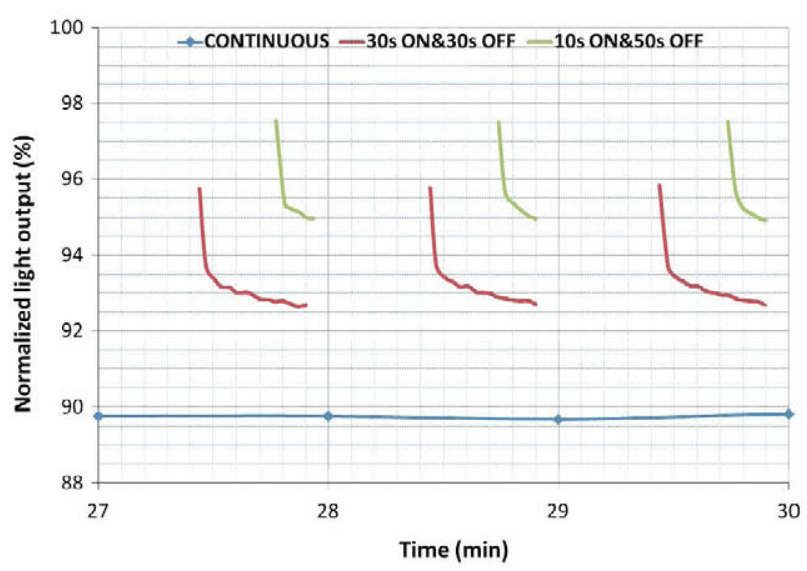

Fig. 5. Detail of normalized light output during stabilized period at three working conditions.

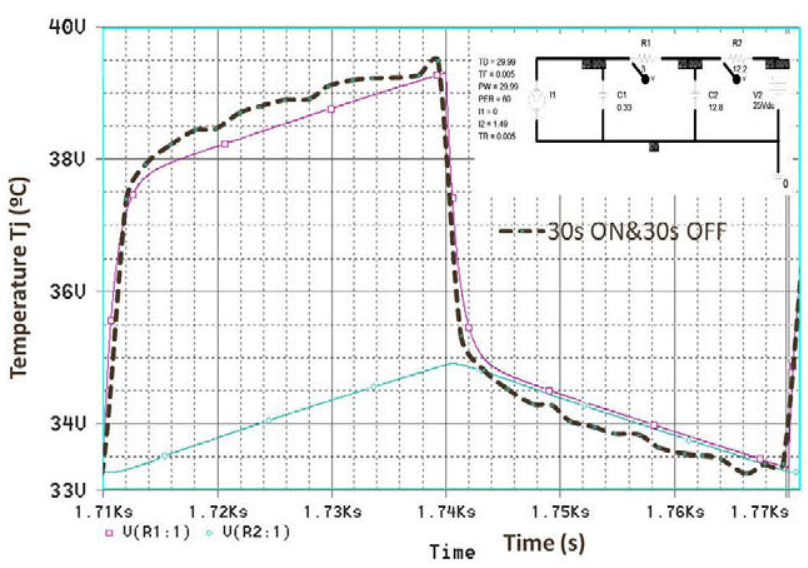

Fig. 6. Experimental junction temperature (dashed line) and PSPICE simulation (continuous lines) for $30 \mathrm{~s} \mathrm{ON}$ and $30 \mathrm{~s}$ OFF condition.

times, $\tau_{1}=R 1 \cdot C 1=1.0 \mathrm{~s}$ and $\tau_{2}=R 2 \cdot C 2=155.6 \mathrm{~s}$ are obtained. Finally, by identifying the value of all parameters, the thermal resistances for the $j-s$ junction to sink and the $s-a$ sink to ambient are: $R 1 \equiv R_{t h j-s}=3{ }^{\circ} \mathrm{C} / \mathrm{W}$ and $R 2 \equiv R_{t h s-a}=12.2^{\circ} \mathrm{C} / \mathrm{W}$. As $\tau=R * C$, the thermal capacitances are: $C 1 \equiv C_{t h j-s}=0.33 \mathrm{~W} \mathrm{~s} /{ }^{\circ} \mathrm{C}$ and $C 2 \equiv C_{\text {th } s-a}=12.8 \mathrm{~W} \mathrm{~s} /{ }^{\circ} \mathrm{C}$.

Simulating with SPICE ${ }^{\mathrm{TM}}$ for the electrical circuit with a voltage of $25 \mathrm{~V}$ (equivalent to Ta) and a cycled current of $1.49 \mathrm{~A}$ (equivalent 
to dissipated power), we obtain the dynamic thermal analysis with a $30 \mathrm{~s} \mathrm{ON}$ and $30 \mathrm{~s}$ OFF condition that fits with the experimental junction temperature in Fig. 6. This model simulates and identifies the dynamic thermal response for other power and cycled conditions.

\section{Light output degradation vs. working condition}

Temperature is an accelerator life stressor [9-13] that can be used to analyze in a short period of time the light output degradation and to identify the device weaknesses from the reliability point of view [19]. Eleven LEDs have been subjected to ALTs (two for each temperature and working condition, except for $90^{\circ} \mathrm{C}$ with only one LED in the cycled condition). The small sample size of these tests will not allow us to obtain accurate reliability data, and therefore, the objective of the tests is to analyze the influence of the working condition in the LED performance evolution.

In this work, two types of failures have been considered: catastrophic failures in which power luminosity decays abruptly to zero and light output degradation failures. Degradation failure is defined in the same way as Alliance for Solid-State Illumination Systems and Technologies (ASSIST) proposes for illumination applications, when light output decays below $70 \%$ of the initial light output [20].

In our tests, we have not observed any catastrophic failures, but several LEDs have failures for the degradation mode. Light output has been assessed by means of the LED light output spectra. The spectra of LEDs are measured periodically during the ALTs outside the ovens at $T_{a}=25 \pm 0.5^{\circ} \mathrm{C}$ with natural convection. The spectra of these LEDs consist of a unique peak at $368 \mathrm{~nm}$ with a full width high maximum (FWHM) of approximately $9 \mathrm{~nm}$. During the tests, we observed a slight peak shift to higher wavelengths $(1.5 \mathrm{~nm})$, but we did not observed any peak widening with an accuracy of $0.3 \mathrm{~nm}$. These values are according to the HP UV-LED manufacturing specifications with a peak wavelength between 365 and $370 \mathrm{~nm}$. Therefore, the peak intensity value has been considered as an assessment of light output performance [9]. All of the LEDs have been characterized with this technique at its initial stage and at different intermediate stages of the test observing a similar behavior for the peak position and FWHM, as shown in Fig. 7.

To evaluate the evolution of the electrical characteristics of LEDs during the ALTs, periodical measurements of the $I-V$ curve outside the ovens at $T_{a}=25 \pm 0.5^{\circ} \mathrm{C}$ and natural convection with a current limit of $1 \mathrm{~A}$ have been developed. In Fig. 8, the $I-V$ curve evolution has been represented for one of the LEDs in the strictest conditions: $90^{\circ} \mathrm{C}$ and continuous mode. As observed, the $I-V$ curve

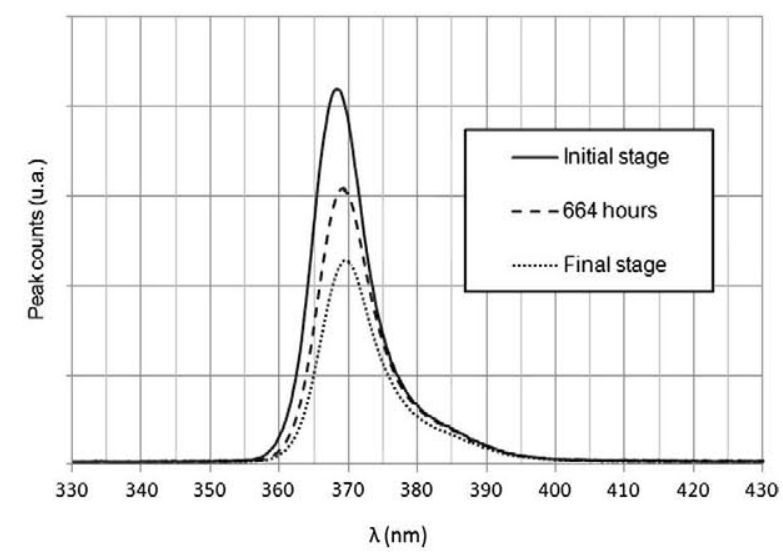

Fig. 7. LED spectrum at different stages of ALT for an LED at $90^{\circ} \mathrm{C}$ and the cycled mode.

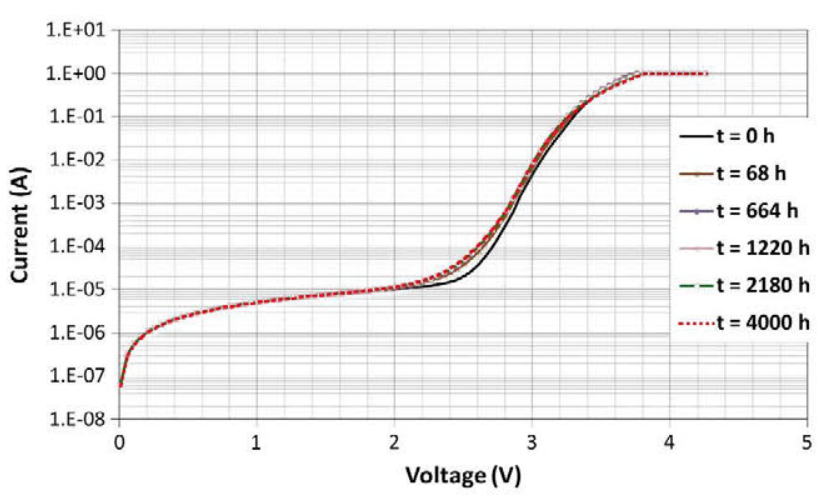

Fig. 8. Evolution of $I-V$ curves for $L E D$ at $90^{\circ} \mathrm{C}$ and in the continuous working condition.

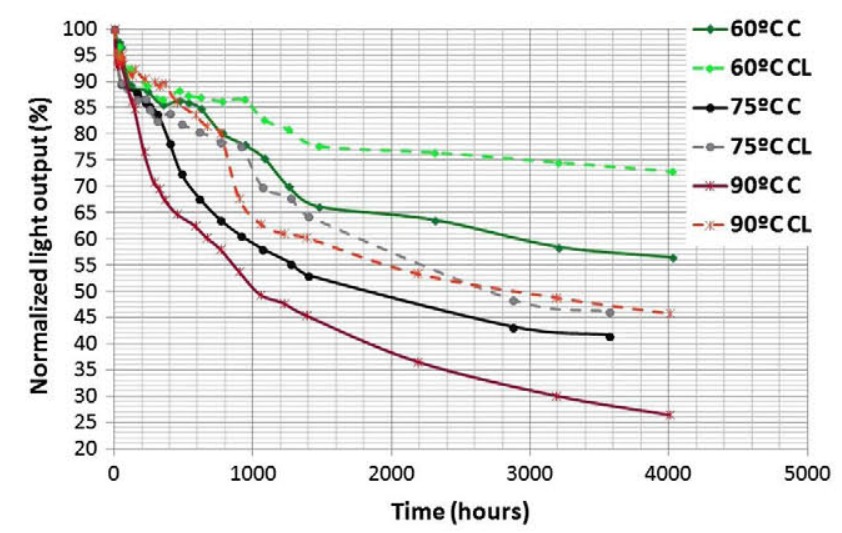

Fig. 9. Normalized light output of six ALTs. The final letter of text box: $\mathrm{C}$ means continuous condition and $\mathrm{Cl}$ means cycled condition.

evolution has two different stages. During the first $68 \mathrm{~h}$, there is a small evolution of the $I-V$ curve that is accomplished with a $12 \%$ power light degradation. After that, from $68 \mathrm{~h}$ until the end of the tests at $4020 \mathrm{~h}$, the evolution of the $I-V$ curve is considerably smaller even though the power light degradation is larger than $82 \%$. All LEDs have a similar $I-V$ curve two-stage evolution and is faster at higher temperatures and in the continuous working mode. During the first tens of hours, there is an evolution at the intermediate voltage of the $I-V$ curve. This period corresponds with power light degradation over $10-20 \%$, and after that, the $I-V$ evolution is considerably lower being power light degradation substantially more important.

In LED reliability analysis [19], there are several mechanisms that simultaneously affect LED degradation. Some of them as semiconductor or wire bonding degradation affect both the $I-V$ curve and power light, but others as package degradation only affect power light degradation. Therefore, it seems that during the initial stage, the predominant degradation appears on semiconductor (wire bonding degradation is reflected at low currents on the $I-V$ curve and is not the case) degradation, and in the second stage, package degradation is the main degradation mechanism. In conclusion, LEDs package degradation is the main failure mechanism.

In Fig. 9, the evolution of the average normalized light output of the LEDs at the different working conditions (C-Continuous and CL-Cycled for $30 \mathrm{~s} \mathrm{ON} / 30 \mathrm{~s} \mathrm{OFF})$ and temperatures $\left(60^{\circ} \mathrm{C}, 75^{\circ} \mathrm{C}\right.$ and $90^{\circ} \mathrm{C}$ ) can be observed. The ALT time for the cycled mode includes the whole cycled time (ON time plus OFF time). From Fig. 9, it is possible to conclude: 
- All of the LEDs have optical power degradation starting at the initial stage.

- Optical power degradation for the same working condition is larger when the ambient temperature is higher.

- Optical power degradation for the same temperature is larger in the continuous working condition than in the cycled working condition.

Considering failure degradation at a $70 \%$ normalized light output in Fig. 9, it can be observed that for the same temperature, the average failure time in the cycled mode is two to three times larger than in the continuous mode.

\section{LEDs package degradation}

LEDs were characterized by optical microscopy to identify package degradation due temperature and working conditions. During the tests, we did not observe any package yellowing, but after several hundreds of hours, we first observed silicone package cracking, and after that, the branched cracks and surface silicone degrade over the chip. In Fig. 10, two optical microscopy photographs of the LED at the initial stage and after cracking are shown. The cracking has an $X$ shape, and it is in line with the two wire bondings of the LED.

Fig. 11 represents the time instant when silicone cracking is detected for the 11 LEDs under test. At higher temperatures and the same working conditions, cracks appear earlier. Furthermore, LEDs working in the continuous working condition have silicone cracking earlier than LEDs working in the cycled condition. Only one LED working in most benign conditions $\left(60^{\circ} \mathrm{C}\right)$ and cycled working conditions does not exhibit silicone cracking during the whole test.

As related to the influence of cracking in light output degradation, in Fig. 12, we represented the normalized light output for LEDs at $60^{\circ} \mathrm{C}$ ALT and the detection time of silicone cracking. It can be observed that LED4 does not have silicone cracking.

We observed in all of the tests (see Fig. 12) that a direct relationship exists between package cracking and an important increase in light output degradation. After the first silicone cracking, the degradation continues with branches and delamination until the degradation failure of the LED. The main conclusion is that silicone package degradation (cracking) is the origin of light output degradation failure.

\section{LED package failure analysis}

The HP UV-LED is an innovative technology in development, especially for deep UV, and it can be assumed that the semiconductor is the weakest element of the device. However, we observed the importance of LEDs package degradation. Although there is an important technological experience in high-power encapsulated

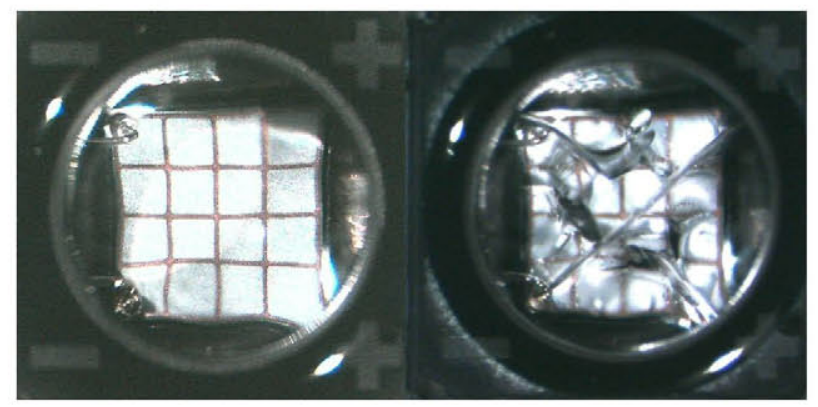

Fig. 10. Optical microscopy of the LEDS at initial stage and after branched cracking.

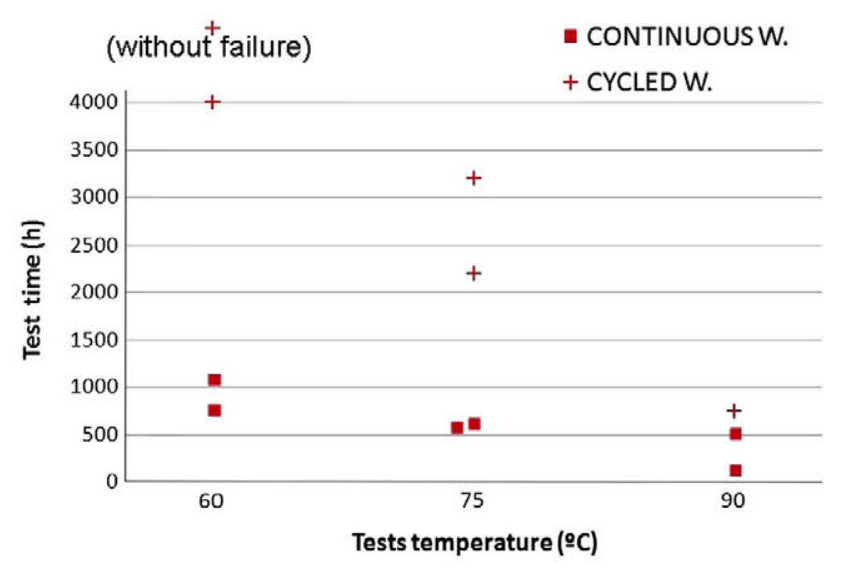

Fig. 11. Test time detection for silicone cracking vs. test temperature for both working conditions.

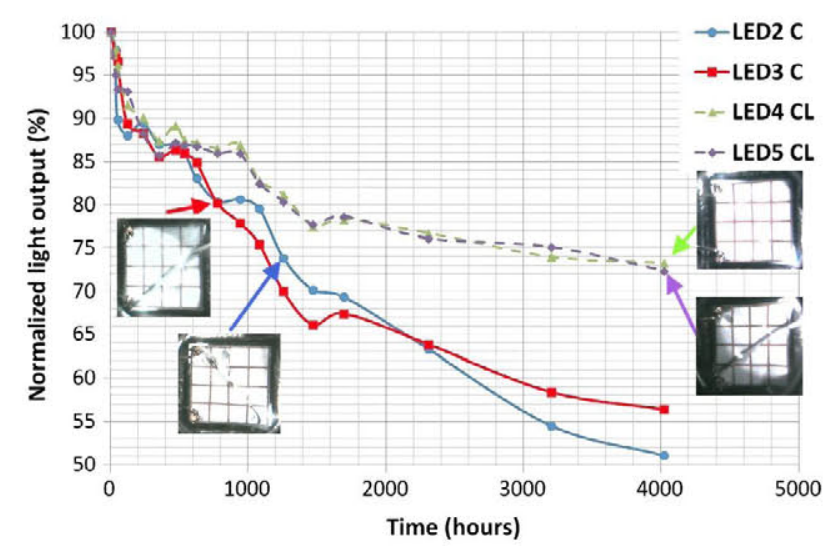

Fig. 12. Normalized light output of the four LEDs at $60^{\circ} \mathrm{C}$ tests and the detection time of silicone cracking. ( $\mathrm{C}$ means continuous condition and $\mathrm{CL}$ means cycled condition).

LEDs [19,21,22], the HP UV-LEDs package has additional stress sources such as high self-heating and high energy UV radiation that must be analyzed.

\subsection{Failure analysis}

As shown in the previous section, in LEDs working with continuous injected current conditions (Figs, 9, 11 and 12), the package degrades earlier than in LEDs working in cycled injected current conditions ( $30 \mathrm{~s} \mathrm{ON}$ and $30 \mathrm{~s} \mathrm{OFF).} \mathrm{Therefore,} \mathrm{temperature} \mathrm{and} \mathrm{con-}$ tinuous time injection cause more package degradation than the thermo-mechanical stress produced by the ON-OFF current injection cycle. Additional LEDs tested at a $90^{\circ} \mathrm{C}$ storage temperature without injection current do not have significant package and light output degradation.

In Fig. 13, the package degradation evolution can be seen for a UV-LED working in continuous mode at $60^{\circ} \mathrm{C}$ ALT. The detection of package degradation occurs at $1080 \mathrm{~h}$ by observing a complete crack that starts in a wire bonding. At $1145 \mathrm{~h}$, a second crack originates in the other wire bonding that evolves at $1215 \mathrm{~h}$. After $1500 \mathrm{~h}$, there are a progressive number of cracks and delaminations (see upper photograph). In the bottom photograph at $1500 \mathrm{~h}$, a detailed LED surface delamination is shown that affects the left side (between the two wire bondings). At $2500 \mathrm{~h}$, in the upper photograph focusing on the glass lens of the LED, it is observed that the crack has broken the silicone located between the chip and the lens. We do not appreciate degradation in any 

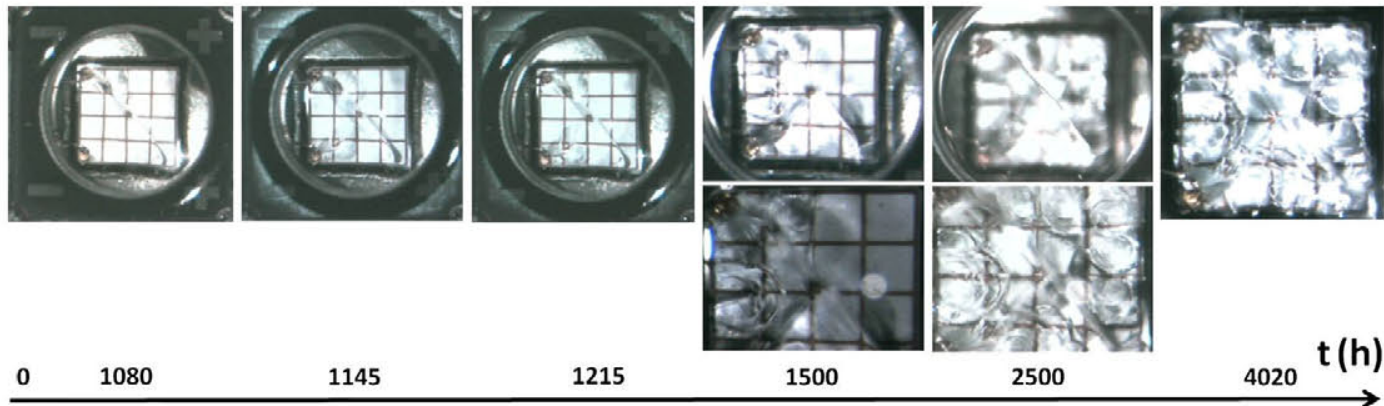

0

1080

1145

1215

1500

2500

$t(h)$

Fig. 13. Photographs of silicone degradation at different instances of time. LED 2 at $60^{\circ} \mathrm{C}$ and continuous working.

glass lens. The detailed photograph at $2500 \mathrm{~h}$ (bottom photograph) focused on the chip, and a delamination with conchoidal shape delimited to metallization can be observed. A photograph at $4020 \mathrm{~h}$ focused on the surface of the chip shows the final state of the LED.

Another relevant result is that silicone degradation produces a self-heating effect due to internal light reflections that originate at a $\mathrm{Tj}$ increment of $1.5^{\circ} \mathrm{C}$ with regard to LEDs without silicone degradation (measured with method from Section 3).

To analyze the LED package degradation process, static thermal simulations have been performed at $T_{a}=25^{\circ} \mathrm{C}$ and $600 \mathrm{~mA}$ continuous working condition. These simulations are based on the $3 \mathrm{D}$ finite element method (FEM), and it is a suitable tool for evaluating package devices and for comparing the experimental results [2326]. In Fig. 14, an FEM thermal simulation of the complete package performed with Autodesk Simulation Multiphysics ${ }^{(B)}$ is shown.

An additional step is to analyze the mechanical stresses due to materials with different coefficients of thermal expansion (CTE) and temperatures. As input parameters, the previous thermal analysis results (Fig. 14) and materials parameters of Table 1 where sapphire is the base material of the UV-LEDs [3], the package has been modeled and simulated with the static stress simulation analysis (Fig. 15). As expected, this simulation shows that the higher stresses are presented in the inter-material bonding, the ball wire bonding with chip, and the ceramic package with $\mathrm{MCPCB}$. However, we have only observed cracks in the silicone package that fills the volume between the chip attached on the ceramic package and the glass lens (shown in the details of Figs. 1 and 15).

In Fig. 15, a detailed von Mises stress analysis can be seen exclusively for the silicone that fills the glass lens. From the simulations, the higher stress in the silicone is close to the two wire bondings

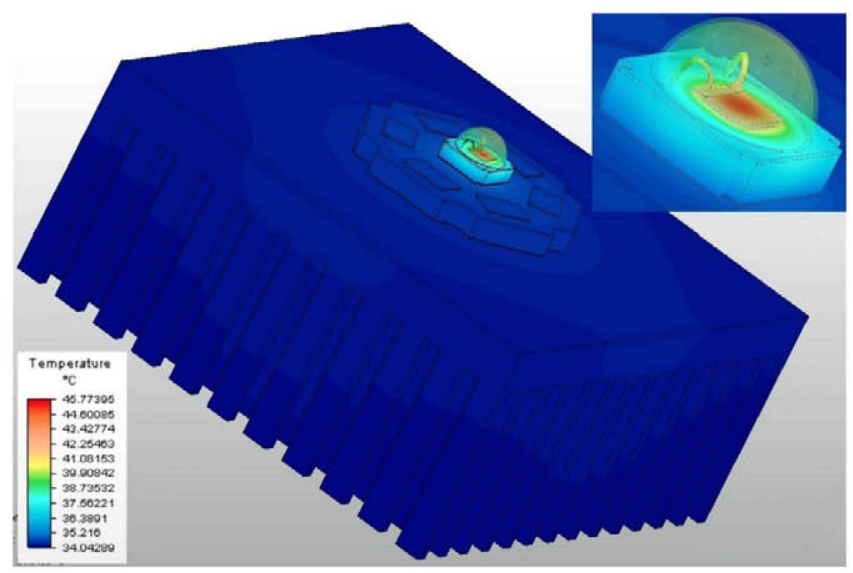

Fig. 14. Static thermal analysis (FEM) of UV-LED at $T_{a}=25^{\circ} \mathrm{C}$ and continuous working condition.
Table 1

Thermal and mechanical properties of upper zone of package.

\begin{tabular}{lllll}
\hline & Sapphire & $\begin{array}{l}\text { Au wire } \\
\text { bonding }\end{array}$ & Silicone & $\begin{array}{l}\text { Glass } \\
\text { lens }\end{array}$ \\
\hline Elastic modulus $(\mathrm{GPa})$ & 400 & 78 & $6.1 \times 10^{-4}$ & 35 \\
Poisson ratio & 0.22 & 0.42 & 0.34 & 0.4 \\
Density $\left(\mathrm{kg} / \mathrm{m}^{3}\right)$ & 3965 & 19,320 & 1200 & 1300 \\
CTE $\left(\mathrm{ppm} /{ }^{\circ} \mathrm{C}\right)$ & 7.9 & 14.2 & 300 & 47 \\
Thermal conductivity & 41.9 & 318 & $1(0.2-$ & 0.58 \\
$\left(\mathrm{~W} / \mathrm{m}^{\circ} \mathrm{C}\right)$ & & & $1.8)$ & \\
\hline
\end{tabular}

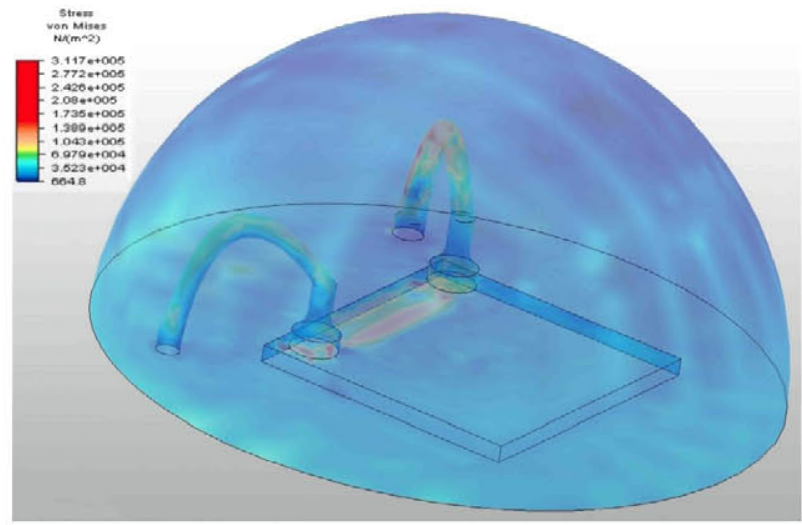

Fig. 15. Results of von Mises static stress analysis model for the silicone that fills the glass lens.

and ball bondings and also to the path between both ball bondings. These simulation stresses are coherent with our experimental results (see Fig. 13), where it can be observed that the silicone crackings originate in the wire bonding. After that, self-heating due the reflection losses in the silicone accelerates the degradation. Additionally, in the first step, new crackings are generated, and in the second step, a delamination of the silicone close to the metallization appears (Fig. 15). The delamination with the shape of "beach markings" (conchoidal fracture) are due to the fatigue of the silicone and arises from thermal expansion mismatches $[27,28]$ between the silicone adhered to the semiconductor chip and the silicone adhered to the upper metallization (Fig. 13).

\subsection{Proposals to mitigate package degradation}

To mitigate package degradation, we propose several solutions in different technical areas. All of them reduce thermomechanical stresses:

Without LED design modifications. It is necessary to look for solutions that achieve less thermal stresses and more homogeneous thermal distribution in the package. There are two possible 
solutions: one for reducing power with the cycled working condition and other for reducing and homogenizing temperature with forced convection by adding a fan to the heat sink.

Replacing the silicone. The optical silicone is presently the best material for UV-LEDs due to its good UV radiation and thermal resistance [19]. However, due to the high CTE of silicone, the interaction of gold wire bonding and the chip with silicone has a complex solution. A possible alternative is to look for silicones with lower CTEs. Another alternative that could solve this problem is to replace the silicone with vacuum or with a gas as nitrogen.

With package modifications. It is necessary to look for package design solutions that reduce the thermo-mechanical stresses between the silicone, chip metallization, and wire bondings. The proposed solutions are the improvement of the chip metallization design [29] and the elimination or modification of wire bonding connections by Through Silicon Via (TSV) technology in the LED chip [29,30]. In any case, a careful analysis considering cost and reliability issues must be developed.

\section{Conclusions}

In this paper, the influence of continuous and cycled working conditions in HP UV-LEDs degradation has been analyzed. Both working modes are compared in ALT tests to obtain results in a suitable period of time. LEDs have been characterized electrically, optically and thermally in static and dynamic conditions.

No catastrophic failure has been observed. Failures are due to normalized light output degradation below $70 \%$. It has been observed that degradation increases with temperature. For the same temperature, the average failure time in cycled mode is two to three times larger than in continuous mode. The main reason is the significant reduction of the Tj LED.

UV-LED parameter evolution during ALTs identifies that the predominant mechanism of failure is encapsulation degradation. From optical microscopy, no encapsulation yellowing has been observed; however, the silicone of the package cracks and the degradation process evolves with the appearance of branches in cracking and the delamination of the silicone with respect chip metallization. This encapsulation failure starts earlier in continuous mode than in cycled mode. In conclusion, silicone package degradation (cracking) is the origin of light output degradation failure.

The encapsulation failure has been analyzed and explained by means of FEM simulations. Based on this analysis, we have proposed a set of possible solutions to mitigate package degradation. All of these alternatives must be analyzed in detail to implement them in the new LEDs package design.

As a main conclusion, we would like to emphasize that cycled working mode conditions improve the long time functionality of HP UV-LEDs. This cycled working condition is suitable for use in many applications such as real-time contaminant fluorescence detection.

\section{Acknowledgments}

The authors would like to thank the Water authority of the Tagus River (Ministry of Agriculture, Food and Environment Spain) for their cooperation. This work was also partially supported by Technical University of Madrid project P125901516.

\section{References}

[1] Lakowicz JR. Principles of fluorescence spectroscopy. 3rd ed. Springer; 2006. http://dx.doi.org/10.1007/978-0-387-46312-4.

[2] Valeur B, Brochon JC, editors. New Trends in fluorescence spectroscopy: applications to chemical and life sciences, vol. 1. Springer; 2001. http: dx.doi.org/10.1007/978-3-642-56853-4.
[3] Muramoto Y, Kimura M, Nouda S. Development and future of ultraviolet lightemitting diodes: UV-LED will replace the UV lamp. Semicond Sci Technol 2014;29(8):084004. http://dx.doi.org/10.1088/0268-1242/29/8/084004.

[4] Arques-Orobon FJ, Gonzalez Posadas V, Jimenez Martin JL, Gutierrez Rios J, Esteban Orobio A. Fluoro-sensing applied to detection and identification of hydrocarbons in inland waters. In: 10th IEEE international conference in networking, sensing and control (ICNSC); 2013. p. 193-8. http://dx.doi.org/10. 1109/ICNSC.2013.6548735.

[5] $\mathrm{Ng} \mathrm{Cl}$, Teo WK, Cai HT, Hemond HF. Characterization and field test of an in situ multi-platform optical sensor. Limnol Oceanogr: Methods 2014;12(7):484-97. http://dx.doi.org/10.4319/lom.2014.12.484.

[6] Mills P, Raymont J. In: kiyoi Ed, editor. The UV-LED. The state of UV-LED curing: an investigation of chemistry and applications. RadTech International; 2013. p. 62.

[7] http://www.bergquistcompany.com/thermal_substrates/LEDs/standardconfigurations.html [9.04.15].

[8] Arques-Orobon FJ, Nuñez N, Vazquez M, González-Posadas V. UV LEDs reliability tests for fluoro-sensing sensor application. Microelectron Reliab 2014;54:2154-8. http://dx.doi.org/10.1016/j.microrel.2014.07.05.9.

[9] Sawyer S, Rumyantsev SL, Shur MS. Degradation of AlGaN-based ultraviolet light emitting diodes. Solid-State Electron 2008;52(6):968-72. http:/ dx.doi.org/10.1016/i.sse.2008.01.027.

[10] Yang L, Hu J, Shin MW. Degradation of high power LEDs at dynamic working conditions. Solid-State Electron 2009;53(6):567-70. http://dx.doi.org/ 10.1016/i.sse.2009.03.014.

[11] Sawant M, Christou A. Failure modes and effects criticality analysis and accelerated life testing of LEDs for medical applications. Solid-State Electron 2012;78:39-45. http://dx.doi.org/10.1016/i.sse.2012.05.042.

[12] Vázquez M, Núñez N, Nogueira E, Borreguero A. Degradation of AllnGaP red LEDs under drive current and temperature accelerated life tests. Microelectron Reliab 2010;50(9):1559-62. http://dx.doi.org/10.1016/j.microrel.2010.07.057.

[13] Núñez N, Vázquez M, González JR, Algora C, Espinet P. Novel accelerated testing method for III-V concentrator solar cells. Microelectron Reliab 2010;50(9):1880-3.

[14] Han-Youl Ryu, Kyoung-Ho Ha, Jung-Hye Chae, Ok-Hyun Nam, Yong-Jo Park. Measurement of junction temperature in GaN-based laser diodes using voltage-temperature characteristics. Appl Phys Lett 2005;87:03506.

[15] Jayawardena A, Liu YW, Narendran N. Analysis of three different junction temperature estimation methods for AC LEDs. Solid-State Electron 2013;86:11-6. http://dx.doi.org/10.1016/i.sse.2013.04.001.

[16] Liu Y, Jayawardena A, Klein TR, Narendran N. Estimating the junction temperature of AC LEDs. In: Tenth international conference on solid state lighting, August 1-5, 2010, San Diego, CA; 2010 proceedings of SPIE 7784: 778409. http://dx.doi.org/10.1117/12.863060.

[17] Shin MW, Jang SH. Thermal analysis of high power LED packages under the alternating current operation. Solid-State Electron 2012;68:48-50. http:II dx.doi.org/10.1016/i.sse. 2011.10.033.

[18] Yang L, Hu J, Shin MW. Dynamic thermal analysis of high-power LEDs at pulse conditions. Electron Dev Lett, IEEE 2008;29(8):863-6. http://dx.doi.org/ 10.1109/LED.2008.2000953.

[19] Chang MH, Das D, Varde PV, Pecht M. Light emitting diodes reliability review. Microelectron Reliab 2012;52(5):762-82. http://dx.doi.org/10.1016/j.microrel. 2011.07.063.

[20] Taylor J. Industry alliance proposes standard definition for LED life LEDs Magazine, April 2005; 2005. p. 9-11.

[21] Lumileds. Luxeon reliability. Reliability datasheet RD25, Philips Lumileds; 2006.

[22] Lin YH, You JP, Lin YC, Tran NT, Shi FG. Development of high-performance optical silicone for the packaging of high-power LEDs. Compon Pack Technol, IEEE Trans 2010;33(4):761-6. http://dx.doi.org/10.1109/TCAPT.2010.2046488.

[23] Yin L, Yang L, Yang W, Guo Y, Ma K, Li S, et al. Thermal design and analysis of multi-chip LED module with ceramic substrate. Solid-State Electron 2010;54(12):1520-4. http://dx.doi.org/10.1016/j.sse.2010.06.028.

[24] Liu S, Liu Y. Modeling and simulation for microelectronic packaging assembly: manufacturing, reliability and testing. John Wiley \& Sons; 2011. http:/l dx.doi.org/10.1002/9780470827826.

[25] Zhang SU, Lee BW. Fatigue life evaluation of wire bonds in LED packages using numerical analysis. Microelectron Reliab 2014. http://dx.doi.org/10.1016/ i.microrel.2014.07.142.

[26] Tan L, Li J, Wang K, Liu S. Effects of defects on the thermal and optical performance of high-brightness light-emitting diodes. Electron Pack Manuf, IEEE Trans 2009;32(4):233-40. http://dx.doi.org/10.1109/TEPM.2009. 2027893.

[27] Lynch SP, Moutsos S. A brief history of fractography. J Fail Anal Prevent 2006:6(6):54-69 +310,1361/154770206X156231.

[28] Bailey C, Tilford T, Ridout S, Lu H. Optimizing the reliability of power electronics module isolation substrates. In: Proceedings of the 2008 international conference on engineering optimization, Rio de Janeiro, Brazil; 2008. p. 1-5.

[29] Hahn B, Galler B, Engl K. Development of high-efficiency and high-power vertical light emitting diodes. Jpn J Appl Phys 2014;53(10):100208. http:/ dx.doi.org/10.7567/ШAP.53.100208.

[30] Van der Plas G, Limaye P, Loi I, Mercha A, Oprins H, Torregiani C, et al. Design issues and considerations for low-cost 3-D TSV IC technology. Solid-State Circ, IEEE J 2011;46(1):293-307. http://dx.doi.org/10.1109/ISSC.2010.2074070. 